\title{
NATURAL HISTORY NOTE \\ Alta longevidad en el murciélago mariposa, Nyctiellus lepidus (Gervais, 1837) (Natalidae), uno de los murciélagos más pequeños del mundo
}

\author{
Humberto Vela Rodríguez ${ }^{1,3}$, Carlos A. Mancina ${ }^{2,3^{*}}$
}

\author{
${ }^{1}$ Grupo Espeleológico Cayo-Barién, \\ Sociedad Espeleológica de Cuba. \\ ${ }^{2}$ Centro Nacional de Biodiversidad, \\ Instituto de Ecología y Sistemática, Cuba. \\ ${ }^{3}$ Programa para la Conservación de los \\ Murciélagos de Cuba (PCMCu). \\ *Corresponding author: \\ mancina@ecologia.cu \\ DOI: https://doi.org/10.14709/ \\ BarbJ.13.1.2020.16
}

Keywords: Antillean bats, caves, Cuba, livespan, longevity quotient, recaptures, roost

Palabras clave: Coeficiente de longevidad, Cuba, cuevas, murciélagos antillanos, refugio, recapturas

received: June, 8th 2020

accepted: October, 10th 2020

\begin{abstract}
RESUMEN
En las últimas décadas se han publicado varios casos de murciélagos que exceden los 20 años de longevidad máxima. La mayoría de éstos se corresponden con especies holárticas que hibernan; sin embargo, datos de longevidad de especies tropicales son aún escasos. En esta nota damos a conocer el primer registro de longevidad para una especie de la familia Natalidae. En este estudio reportamos la recaptura de un macho de Nyctiellus lepidus que había sido marcado 16,9 años antes en la misma cueva en la región central de Cuba. Esta especie es endémica de Cuba (incluyendo la Isla de la Juventud) y las Bahamas, y se refugia exclusivamente en cuevas. Nyctiellus lepidus tiene una masa corporal de aproximadamente $2 \mathrm{~g}$, lo que lo ubica entre los murciélagos más pequeños del mundo. Basado en el coeficiente de longevidad, la proporción entre la longevidad observada y la esperada, se estimó que puede vivir 3,9 veces de lo esperado para su masa corporal. Este registro de longevidad constituye uno de los más largos para murciélagos insectívoros no hibernantes.
\end{abstract}

\begin{abstract}
Over the last two decades, there have been several longevity records of bats that have exceeded 20 years, mostly corresponding to Holarctic hibernating species. Whereas such data of tropical bats is still scarce. Here we report the first longevity record for the Gervais's funnel-eared bat, Nyctiellus lepidus (Gervais, 1837) (family Natalidae). We recaptured a male individual that had been banded 16.9 years previously in the same cave in Central Cuba. This species is endemic to Cuba (including Isla de la Juventud) and the Bahamas and is an obligate cave-dwelling bat. Weighing nearly 2 grams, N. Lepidus is one of the world's smallest bats. Based on longevity quotient (the ratio of observed to predicted longevity), we estimate that its lifespan is 3.9 times longer than expected for its body mass. This longevity record constitutes one of the longest in a non-hibernating insectivorous bat.
\end{abstract}

\section{INTRODUCCIÓN}

La longevidad es un atributo relacionado con la historia de vida de la especie, y en teoría el valor promedio de la longevidad en una población se incrementa a medida que decrece la probabilidad de muerte, debido a factores extrínsecos, como la depredación y las enfermedades infecciosas (Austad \& Fischer 1991, Carey 2003). Dentro de los mamíferos, los murciélagos se encuentran entre los de mayor longevidad; de hecho, la longevidad de los murciélagos es 3,5 veces mayor que la de cualquier mamífero placentario no volador de similar masa corporal (Wilkinson \& South 2002). Comparado con otros mamíferos, la capacidad de volar de los murciélagos, así como su tolerancia a ciertos patógenos, podrían reducir riesgos de mortalidad (MunshiSouth \& Wilkinson 2010, Kacprzyk et al. 2017).

En la actualidad el récord de máxima longevidad para un murciélago corresponde a un individuo de Myotis brandtii que fue recapturado 41 años después de haber sido marcado en una región de Siberia, Rusia (Podlutsky et al. 2005); no obstante, existen varios registros de más de 30 años (ej. Davis \& Hitchcock 1995, Arlettaz et al. 2002) y en todos los casos se corresponden a especies de ambientes templados que hibernan. El conocimiento sobre la longevidad en los murciélagos se ha incrementado debido al aumento de los estudios que involucran el marcaje de los individuos; no obstante, los datos son comparativamente escasos para murciélagos tropicales (Wilkinson \& Adams 2019). En esta nota, por primera vez se brindan datos sobre la longevidad en una especie de la familia Natalidae, el murciélago mariposa, Nyctiellus lepidus (Gervais, 1837) (Fig. 1).

La familia Natalidae es endémica del Neotrópico e incluye 12 especies de hábitos insectívoros que se especializan en el uso de las cuevas como refugio diurno (Tejedor 2011). En particular, Nyctiellus lepidus, se distribuye en Cuba, Isla de la Juventud y algunas islas de Las Bahamas; utiliza como refugio salones o galerías que posibiliten la creación de 


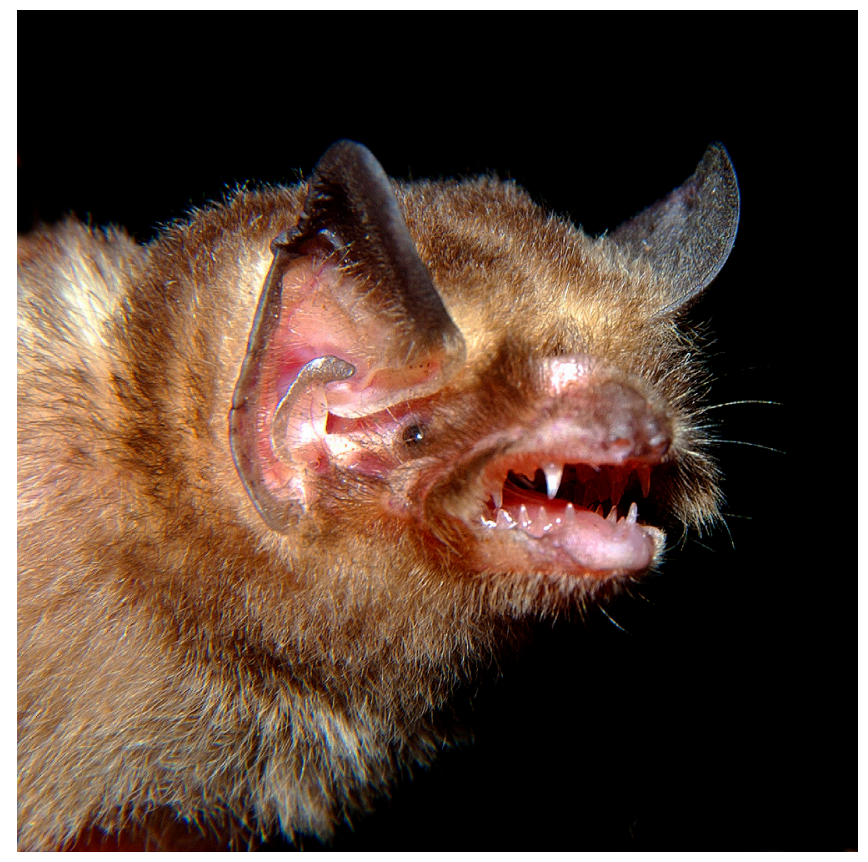

Fig. 1 - Macho adulto de murciélago mariposa, Nyctiellus lepidus (foto: Carlos A. Mancina). // Adult male of Gervais's funnel-eared bat, Nyctiellus lepidus (photo: Carlos A. Mancina).

un ambiente cálido y húmedo, donde forman colonias que pueden alcanzar varios miles de individuos (Silva Taboada 1979). Con una masa corporal entre 1,9 y $2,7 \mathrm{~g}$. (Silva Toboada 1979), N. lepidus es la especie más pequeña de los natálidos y uno de los murciélagos más pequeños del mundo, solo comparado con Craseonycteris thonglongyai ( 2,0 g) y Pipistrellus nanulus ( 2,5 g) (Hill \& Smith 1981); sin embargo, de estas especies no existe información sobre su longevidad.

\section{MATERIALES Y MÉTODOS}

Los datos fueron obtenidos de un monitoreo a largo plazo (entre los años 1995 y 2018) de las poblaciones de Nyctiellus lepidus que habitan las cuevas al norte de las provincias Las Villas, Sancti Spíritus y Ciego de Ávila, en la región central de Cuba. Entre los objetivos de este estudio se encontraban, la estimación de los tamaños poblacionales mediante métodos de marcaje y recaptura, así como conocer los movimientos locales entre cuevas, asociados al periodo reproductivo y la fidelidad a los refugios. Para eso, en el año 1995, se realizó el marcaje de 2494 individuos adultos (57,1\% 오; 42,9\% $\hat{\jmath}$ ) en las cuevas: Guajabana II (municipio Caibarién, -79.458; 22.453), Rodolfo (Yaguajay, -79.387; 22.406) y la Tapia (Remedios, -79.530; 22.471). Los murciélagos fueron capturados con redes manuales dentro de las cuevas y se les colocó una anilla numerada en el antebrazo. Las anillas se confeccionaron de aluminio ligero y fueron rotuladas con códigos grabados a mano que permitían la identificación individual. Los murciélagos marcados fueron liberados en el mismo lugar de captura. Posteriormente, entre los años 1999 y 2018 , se realizaron capturas, tanto en las cuevas donde se realizaron los marcajes, como otras de la región que son utilizadas como refugio diurno por la especie. Información suplementaria sobre este estudio se puede encontrar en Vela Rodríguez et al. (2019).
Para ilustrar el valor de longevidad observada en $N$. lepidus, respecto a otras especies de murciélagos, se realizó una regresión entre la masa corporal y la longevidad máxima observada en otras 59 especies de microquirópteros de hábitos insectívoros (Material Suplementariao 1), incluyendo hibernantes y no hibernantes; los datos fueron extraídos de Wilkinson \& Adams (2019) (http://hdl.handle. net/1903/21501). Dado que en los mamíferos existe relación alométrica entre la masa corporal y la longevidad (Austad \& Fischer 1991), es necesario eliminar el efecto de la masa para realizar comparaciones entre $N$. lepidus y otras especies. Para esto, se calcularon los Coeficientes de Longevidad (LQ), que es la proporción entre la longevidad observada en la especie y la esperada para su masa corporal. La longevidad esperada fue calculada usando la ecuación: $\log _{10}($ longevidad $)=0,5609+\log _{10}($ masa corporal $) \times 0,1868$, derivada de la regresión entre la longevidad y la masa del cuerpo de 804 mamíferos placentarios no voladores (Wilkinson \& Adams 2019). La masa corporal utilizada para $N$. lepidus fue de $2,5 \mathrm{~g}$, valor obtenido de la masa promedio de 1104 machos de las poblaciones que habitan la región central de Cuba.

\section{RESULTADOS Y DISCUSIÓN}

Del total de individuos marcados en 1995, posteriormente al año 1999 sólo se recapturaron 36 (18 hembras y 18 machos), lo que representa $1,4 \%$ de recapturas (Tabla 1). Entre los períodos siguientes 2004-2006, 2008-2010 y 2012-2018 no se obtuvieron recapturas. De los individuos recapturados, 18 tenían más de 6 años de edad. En el año 2007 se recapturó en cueva Rodolfo una hembra marcada 4318 días (11,8 años) antes en cueva la Tapia, ambas cuevas separadas por $15,5 \mathrm{~km}$. Este dato, además de mostrar que la especie se familiariza con áreas más amplias de las que previamente se habían planteado (ver Vela Rodríguez et al. 2019), sugiere la existencia de fidelidad de las hembras por las cuevas utilizada como colonias de maternidad, como es el caso de cueva Rodolfo. En el año 2011 en la cueva Guajabana II se recapturaron dos machos que habían sido marcados 6150 días (16,8 años) y 6203 (17 años) antes, en la misma cueva. Es importante señalar que ambos eran adultos al momento de ser marcados, por lo que la edad de estos dos machos es mayor que la estimada por el tiempo transcurrido desde sus recapturas. Ninguno de los individuos mostró daños relacionados con el anillamiento.

Los valores de longevidad máxima observados en $N$. lepidus permiten expandir el rango de longevidad conocido para los murciélagos, ya que se ubican en el límite inferior de la masa corporal del orden. Comparado con los del resto de los murciélagos insectívoros no hibernantes, $N$. lepidus se encuentra entre los de mayor longevidad (Fig. 2). Al contrastar los datos con los de murciélagos neotropicales de la familia Phyllostomidae, los valores de $N$. lepidus son comparables a los más altos registrados (ver Material Suplementario 1). Solo en tres especies se registran mayores valores de longevidad: Phyllostomus hastatus (22 años), Artibeus jamaicensis (19,2 años) y Desmodus rotundus (29,2 años); no obstante, en las dos últimas especies los datos provienen del cautiverio, donde la expectativa de vida aumenta al reducirse los riesgos de mortalidad. 
Tabla 1 - Número de murciélagos capturados entre 1999 y 2011, incluyendo las recapturas ( $\hat{0} \hat{\delta} / 0$ 우) de los marcados en 1995 y la edad mínima de estas recapturas // Number of captures between 1999 and 2011 and recaptured ( $\hat{\alpha} \delta / O+q)$ obtained in successive years.

\begin{tabular}{lccccccc} 
& $\mathbf{1 0}$ Año \\
\cline { 2 - 8 } & $\mathbf{1 9 9 9}$ & $\mathbf{2 0 0 0}$ & $\mathbf{2 0 0 1}$ & $\mathbf{2 0 0 2}$ & $\mathbf{2 0 0 3}$ & $\mathbf{2 0 0 7}$ & $\mathbf{2 0 1 1}$ \\
\hline No. capturas & 492 & 1431 & 1134 & 2050 & 1969 & 1170 & 1384 \\
Recapturas (M/H) & $5(3 / 2)$ & $9(6 / 3)$ & $4(0 / 4)$ & $10(4 / 6)$ & $5(3 / 2)$ & $1(0 / 1)$ & $2(2 / 0)$ \\
Edad mínima (años) & $3,2-3,9$ & $4,1-4,8$ & $5,3-5,9$ & $6,0-7,0$ & $7,2-7,6$ & 11,8 & $16,8-17,0$ \\
\hline
\end{tabular}

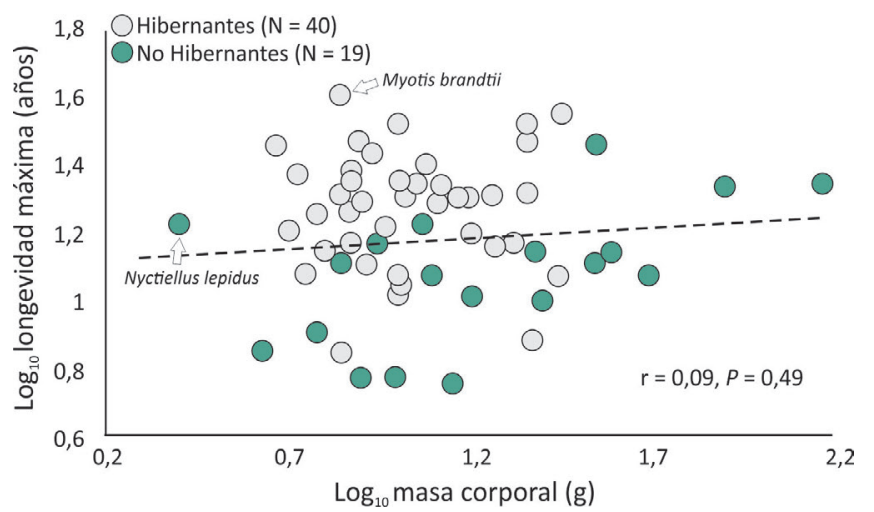

Fig. 2 - Relación entre la longevidad y la masa corporal en 59 especies de murciélagos insectívoros. Las flechas indican a Nyctiellus lepidus (Natalidae) y Myotis brandtii (Vespertilionidae), este último presenta el record de longevidad en murciélagos. // Relation between longevity and body mass in 59 insectivorous bat species. The arrows indicate to Nyctiellus lepidus (Natalidae) and Myotis brandtii (Vespertilionidae), this last with longest longevity record in bats.

El Coeficiente de Longevidad calculado para N. lepidus fue de 3,91 , lo que indica que la longevidad observada supera en 3,9 veces la esperada para un mamífero de similar masa. Este valor de LQ es superior al promedio de los murciélagos, que es de 2,67 , calculado a partir de las 91 especies disponibles en Wilkinson \& Adams (2019) y el mayor conocido para insectívoros no hibernantes (ver Material Suplementario 1). Según estos autores, especies con $\mathrm{LQ}>$ 4,2 pudieran ser consideradas de "longevidad extrema", y se presenta en unas pocas especies hibernantes de las familias Vespertilionidae y Rhinolophidae, así como en D. rotundus. Wilkinson \& Adams (2019), encontraron que la masa corporal y la conducta de hibernación son las variables que mejor predicen la longevidad en los murciélagos. Sin embargo, cuando analizaron exclusivamente especies que no hibernan, observaron que la ausencia de dimorfismo sexual y el uso de cuevas como refugio son las variables que mejor predicen la longevidad. No obstante, $N$. lepidus es una de las especies más pequeñas del orden y presenta dimorfismo sexual secundario, donde las hembras son mayores que los machos (Silva Taboada 1979). Similar a otras especies (Podlutsky et al. 2005, lbáñez et al. 2018), la mayor longevidad se observó en individuos machos. Esto podría estar relacionado con la conducta más sedentaria de los machos. Durante el período reproductivo las hembras de $N$. lepidus realizan migraciones locales y regionales hacia cuevas que son utilizadas como colonias de maternidad (Vela Rodríguez et al. 2019), lo que podría incrementar el riesgo de depredación.

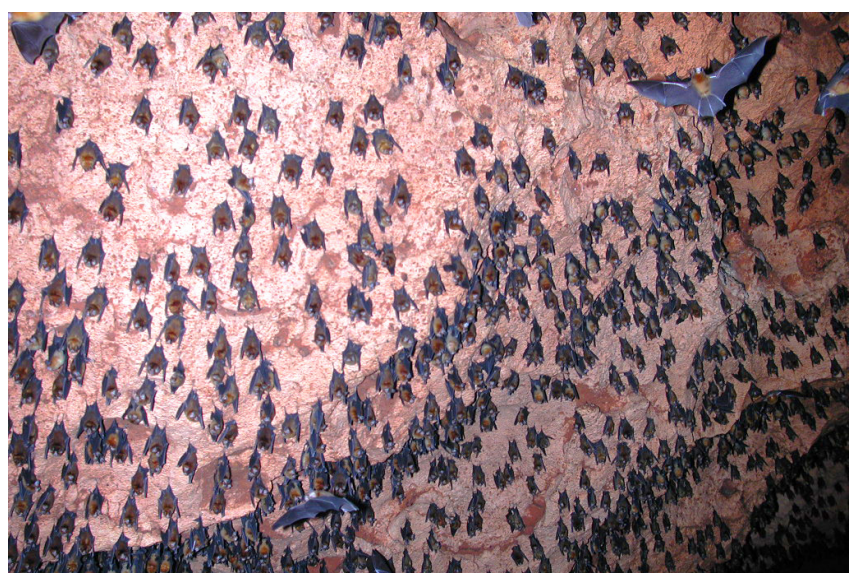

Fig. 3 - Grupo de Nyctiellus lepidus refugiándose en una cueva de Cuba central. Esta especie generalmente no comparte los salones cavernarios con otras especies, y los individuos descansan separados unos de otros; esta conducta podría reducir la transmisión de parásitos y patógenos (foto: Abel Hernández). // Cluster of Nyctiellus lepidus roosting in a cave of Central Cuba. This species usually does not share the cave rooms with other species, and the individuals roost uniformly spaced out; this behavior could reduce the intraspecific transmission of parasites and pathogens (photo: Abel Hernández).

Aunque en Cuba se han realizado otros estudios de monitoreo a largo plazo de murciélagos que involucran el marcaje de los individuos (ej. Mancina 2011), los datos sobre la longevidad en otras especies están limitados a muy escasos eventos de recapturas, y no exceden los ocho años (Mancina obs. pers.). No obstante, comparado con otras especies que habitan el archipiélago cubano, existen varios factores que podrían explicar los altos valores de longevidad observada en $N$. lepidus. A diferencia de otros murciélagos insectívoros, $N$. lepidus no comparte los salones cavernarios con otras especies y los individuos descansan separados unos de otros (Silva Taboada 1979) (Fig. 3), lo que podría reducir la transmisión inter e intraespecífica de parásitos y patógenos. Observaciones realizadas por Silva Taboada (1979) indican que, a diferencia de otras especies, $N$. lepidus no muestra una conducta letárgica ante las bajas temperaturas; sin embargo, exhibe cierta labilidad térmica. Como sugieren Wilkinson \& Adams (2019), la capacidad de tolerar un amplio rango de temperaturas corporales, pudiera ser un factor importante para sobrevivir a infecciones virales u otros patógenos. Debido a que aún son limitados los datos sobre longevidad en murciélagos, y particularmente en especies tropicales, consideramos que los datos obtenidos para N. lepidus podrían ser importantes para futuros estudios relacionados con el comportamiento de la longevidad en los mamíferos y particularmente en murciélagos. 


\section{AGRADECIMIENTOS}

A todos los amigos que durante años han apoyado el trabajo de campo, en particular a Francisca Amador, Martin Núñez y Javier Pérez. A los colegas del Parque Nacional Caguanes, en especial a Armando Falcón y Miguel A. Delgado, por su constante apoyo. Los comentarios aportados por dos revisores anónimos contribuyeron a mejorar la versión inicial del manuscrito.

\section{REFERENCIAS}

ARLETTAZ, R., CHRISTE, P. \& DESFAYES, M. (2002). 33 years, a new longevity record for a European bat. Mammalia, 66(3): 441-442.

AUSTAD, S. N. \& FISCHER, K. E. (1991). Mammalian Aging, Metabolism, and Ecology: Evidence from the bats and marsupials. J Gerontol, 46(2): B47-B53. https://doi. org/10.1093/geronj/46.2.B47

CAREY, J. R. (2003). Life Span: A conceptual overview. Popul Dev Rev, 29: 1-18.

DAVIS W. H. \& HITCHCOCK H. B. (1995). A new longevity record for the bat Myotis lucifugus. Bat Research News, 36: 6.

HILL, J. E. \& SMITH, S. E. (1981). Craseonycteris thonglongyai. Mammalian Species, 160(3): 1-4. https://doi. org/10.2307/3503984

IBÁÑEZ, C., NOVELLA-FERNANDEZ, R., ALONSO, P. \& AGIRRE-MENDI, P. T. (2018). New longevity record for the Mediterranean horseshoe bat (Rhinolophus euryale Blasius, 1853). JBRC, 11(1): 80-82. http://doi. org/10.14709/BarbJ.11.1.2018.09

KACPRZYK, J., HUGHES, G. M., PALSSON-MCDERMOTT, E. M., QUINN, S. R., PUECHMAILLE, S. J., O'NEILL, L. A. J. \& TEELING, E. C. (2017). A Potent Anti-Inflammatory Response in Bat Macrophages May Be Linked to Extended Longevity and Viral Tolerance. Acta Chiropterol, 19(2): 219-228. http://doi.org/10.3161/15081109A CC2017.19.2.001
MANCINA, C. A. (2011). Los murciélagos de la Reserva de la Biosfera "Sierra del Rosario", Cuba: un proyecto de monitoreo a largo plazo. Bol Red Latin Cons Murc, 2(4): 5-9.

MUNSHI-SOUTH, J. \& WILKINSON, G. S. (2010). Bats and birds: Exceptional longevity despite high metabolic rates. Ageing Res Rev, 9(1): 12-19. http://doi.org/10.1016/j. arr.2009.07.006

PODLUTSKY, A. J., KHRITANKOV, A. M., OVODOV, N. D. \& AUSTAD, S. N. (2005). A new field record for bat longevity. The Journals of Gerontology: Series A, 60(11): 1366-1368. https://doi.org/10.1093/gerona/60.11.1366

SILVA TABOADA, G. (1979). Los Murciélagos de Cuba. ed.: Editorial Academia. La Habana, Cuba, 423 pp.

TEJEDOR, A. (2011). Systematics of Funnel-Eared Bats (Chiroptera: Natalidae). B Am Mus Nat Hist, 2011(353): 1-140. https://doi.org/10.1206/636.1

VELA RODRÍGUEZ, H., AMADOR HERNÁNDEZ, F., NÚÑEZ RODRÍGUEZ, M. \& PÉREZ PARET, J. (2019). Notas sobre la conducta reproductiva del murciélago mariposa, Nyctiellus lepidus (Chiroptera: Natalidae) en cuevas al Norte de la región central de Cuba. Poeyana, 508:12-17.

WILKINSON, G. S. \& SOUTH, J. M. (2002). Life history, ecology and longevity in bats. Aging Cell, 1(2): 124-131. https:// doi.org/10.1046/j.1474-9728.2002.00020.x

WILKINSON, G. S. \& ADAMS, D. M. (2019). Recurrent evolution of extreme longevity in bats. Biol Letters, 15(4): 20180860. https://doi.org/10.1098/rsbl.2018.0860 